$\begin{array}{lllllllllllllll}\text { A C T A } & \text { C H E M I C A } & \text { S C A N D I N A V I C A } & 13 & \text { (1959) } & 409-414\end{array}$

\title{
The Degradation Product from Male Sperm Antagglutin with Molten Potassium Bisulphate
}

\author{
SVANTE ROSS
}

Institute of Zoophysiology, University of Uppsala, Sweden

\begin{abstract}
The degradation product from male sperm antagglutin with molten potassium bisulphate has been compared with the corresponding products from some model compounds, especially tocopherols and hydroquinone. Some reactions taking place in the molten potassium bisulphate have been studied. The chemical structure of the antagglutin product has been discussed.
\end{abstract}

$\mathrm{M}_{\mathrm{s} \cdot \operatorname{sen}}$

ale sperm antagglutin, a substance which in mammalian spermatozoa ${ }^{1,2}$, prevents head-to-head agglutination, is a proteid containing sugar ${ }^{2}$, sulphuric acid residues ${ }^{3}$, and a further component which, probably in a somewhat modified form has been sporadically extracted with ethyl ether from antagglutin preparations. Judging from its ultra-violet spectrum this extractable artefact seems to be an aromatic compound of diphenolic character. It is easily oxidized to a quinone-like substance ${ }^{4,5}$.

Heating of male sperm antagglutin in molten potassium bisulphate at $220^{\circ} \mathrm{C}$ gives a product which is soluble in ethanol and water, and which after oxidation shows a rather high optical density in ultra-violet light with a maximum at $256 \mathrm{~m} \mu$. The transformation of the antagglutin into this oxidized compound has been used as a method for quantitative determination of male sperm antagglutin ${ }^{6}$. This oxidized product is very similar to the oxidized form of the above mentioned aromatic substance which was extracted from the antagglutin solutions. As this degraded substance is rather easily formed under controlled conditions, experiments have been performed in order to obtain information about its properties and its precursor in the antagglutin molecule. Unfortunately the formed product is very easily further oxidized and therefore difficult to isolate. For this reason some model substances have been treated in the same way as the antagglutin, and the properties of the obtained products compared with those of the product from antagglutin. Some information about the chemical reactions that may occur in the molten potassium bisulphate have also been obtained from this model experiments. 


\section{EXPERIMENTAL AND RESULTS}

The treatment of the male sperm antagglutin and the model substances with'molten potassium bisulphate has been performed according to the method of quantitative determination of male sperm antagglutin ', but with some modifications: about $1-3 \mu \mathrm{g}$ male sperm antagglutin or $0.1-1 \mathrm{mg}$ of the model substance is mixed with a large quantity $(1-2 \mathrm{~g})$ of dried, ether-extracted potassium bisulphate. This is done in a test tube with a diameter of one $\mathrm{cm}$ and a length of six $\mathrm{cm}$. This reaction tube is placed in a bigger tube in which it is held upright by a layer of glass beads at the bottom. The bigger tube is immersed for one hour into an oil bath of $220^{\circ} \mathrm{C}$. After cooling the reaction tube is cleaned externally with ethyl ether, and then reduced to powder in a mortar together with its contents. The powder is extracted with $3 \mathrm{ml}$ of absolute ethanol in a centrifuge tube with a glass stopper. The centrifugated ethanol extract is evaporated under vacuum at $50^{\circ} \mathrm{C}$, and the residue extracted several times with $3 \mathrm{ml}$ of freshly distilled, dried ethyl ether. The residue in the distillation flask is taken up in $3 \mathrm{ml}$ of absolute ethanol or distilled water, and its absorption spectrum in ultra-violet light read, especially between 250 and $260 \mathrm{~m} \mu$, where the degradation product from male sperm antagglutin has a maximum at $256 \mathrm{~m} \mu$. In some cases manganese dicxide is added to the powder during the extraction in order to act as an oxidizing agent, but in other cases the oxidation is performed through aeration in presence of platinum black. A Beckmann DU spectrophotometer was used for the absorption readings.

Hydroquinone disulphonic acid has been synthetized according to v. Hesse ?. The identity and purity of the obtained product was acertained spectrophotometrically. The other chemicals used are commercial preparations of pro analysi purity.

Besides the male sperm antagglutin only few of the tested compounds give products after treatment with molten potassium bisulphate which have absorption maxima at $256 \mathrm{~m} \mu$ (Table 1), viz. $\alpha$-and $\delta$-tocopherol on the one side, and hydroquinone and some substances which in molten potassium bisulphate may be transformed into hydroquinone, on the other.

The absorption maximum at $256 \mathrm{~m} \mu$ of the degradation product from male sperm antagglutin disappears, when the solution is made alkaline, but reappears again when the solution is acidified (Fig. 1). The absorption maximum also disappears after hydrogenation with hydrogen and a quantity of platinum black, but reappears after aeration of this solution. The compound with absorption maximum at $256 \mathrm{~m} \mu$ is very easily oxidized, and the maximum is then

Table 1. Compounds which have been tested with reference to the absorption maximum at $256 \mathrm{~m} \mu$ after treatment with molten potassium bisulphate.

Aromatic compounds.

Phenol

Hydroquinone

Pyrocatechol

Resorcinol

Durohydroquinone

Phloroglucinol

$p$-Benzoquinone

2,5-Dihydroxybenzoio acid

$p$-Dimethoxybenzene

$a$-Tocopherol

$\delta$-Tocopherol

Tyrosine

Tryptophane

Coumarine
Aliphatic compounds.
Quinic acid

Inositol

1,4-Cyclohexadion

Glucose

Fructose

Arabinose 


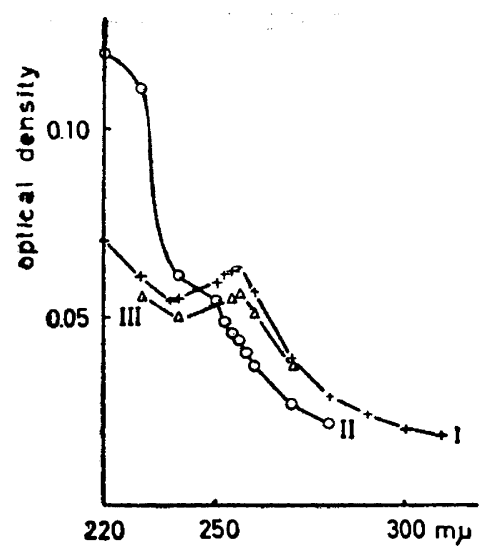

Fig. 1. The absorption in ultra-violet light of the degradation product of male sperm antagglutin with molten potassium bisulphate. I, pH about 4; II, pH about 12; III, $\mathrm{pH}$ restored to 4 . Solvent distilled water.

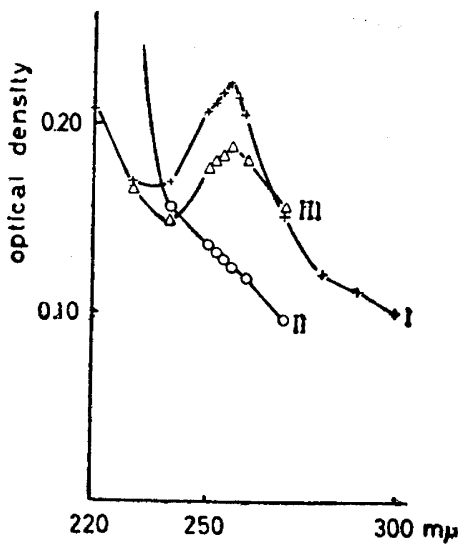

Fig. 2. The absorption in ultra-violet light of the product of $\alpha$-tocopherylphosphete and molten potassium bisulphate. I, $\mathrm{pH}$ about 4; II, pH about 12; III, pH restored to 4. Solvent distilled water.

irreversibly lost. The degraded product from antagglutin is soluble in water and ethanol, but not in ethyl ether (Table 2).

The products from $\alpha$-tocopherol and its acetate and phosphate-esters and $\delta$-tocopherol after treatment with molten potassium bisulphate have the same properties as those of the degradation product from male sperm antagglutin (Fig. 2 and Table 2). The yields of the products are, however, very poor, only about one hundreth $(\mathrm{w} / \mathrm{w})$ of that of the degraded product from antagglutin. Trials to isolate the product from $\alpha$-tocopherol have failed owing to its sensitivity to further oxidation.

From Table 1 it is obvious that hydroquinone as well as compounds that in molten potassium bisulphate may be transformed into hydroquinone, like quinic acid, inositol, and 2,5-dihydroxybenzoic acid, give a product with an absorption maximum at $256 \mathrm{~m} \mu$ (Fig. 3:I). With these compounds also a maximum at $312 \mathrm{~m} \mu$ is obtained which has never been observed with the products from antagglutin or the tocopherols. This absorption maximum at $312 \mathrm{~m} \mu$ is identical with that of hydroquinone disulphonic acid (Fig. 3:II) which after oxidation with manganese dioxide in $80 \%$ ethanol gives a compound with an absorption maximum at $256 \mathrm{~m} \mu$ (Fig. 3:III).

This compound is $p$-benzoquinone disulphonic acid, the dipotassium salt of which in aqueous solution at $\mathrm{pH} 4$ has a molar extinction at $256 \mathrm{~m} \mu$ of about 14500 . Hydroquinone is hence sulphonated in the molten potassium bisulphate which explains the non-solubility of the reaction product in ethyl ether (Table 2).

The product obtained from hydroquinone, although not identical with the product obtained from antagglutin, shows qualities which are similar to those of the latter (Table 2). Thus the absorption maximum at $256 \mathrm{~m} \mu$ disappears,

Acta Chem. Scand. 13 (1959) No. 3 
Table 2. Some properties of the compounds that give products with an absorption maximum at 256 m $\mu$ after treatment with molten potassium bisulphate.

\begin{tabular}{|c|c|c|c|c|c|c|c|c|}
\hline \multicolumn{3}{|c|}{ Before treatment } & \multicolumn{6}{|c|}{ After treatment } \\
\hline Compound & $\begin{array}{l}\text { Ab- } \\
\text { sorp- } \\
\text { tion } \\
\text { maxi- } \\
\text { mum }\end{array}$ & Solubility & $\begin{array}{l}\text { Ab- } \\
\text { sorp- } \\
\text { tion } \\
\text { maxi- } \\
\text { mum }\end{array}$ & \begin{tabular}{|c|} 
Ab- \\
sorp- \\
tion \\
maxi- \\
mum \\
after \\
reduction
\end{tabular} & $\begin{array}{l}\text { The pH. } \\
\text { dependence } \\
\text { of the } \\
\text { absorption } \\
\text { maximum }\end{array}$ & Solubility & $\begin{array}{l}\text { Oxida- } \\
\text { bility }\end{array}$ & $\begin{array}{l}\text { The } \\
\text { resulting } \\
\text { product }\end{array}$ \\
\hline $\begin{array}{l}\text { rMale sperm } \\
\text { antagglutin } \\
\mathbf{r a}_{\alpha \text {-Tocopherol }} \\
\text { ס-Tocopherol } \\
\text { InHydroquinone }\end{array}$ & $\begin{array}{l}292 \mathrm{~m} \mu \\
297 \mathrm{~m} \mu \\
290 \mathrm{~m} \mu\end{array}$ & $\begin{array}{l}\text { Ethanol, ether } \\
\text { Ethanol, ether } \\
\text { Ethanol, ether }\end{array}$ & $256 \mathrm{~m} \mu$ & $312 \mathrm{~m} \mu$ & $\begin{array}{c}\text { The maximum } \\
\text { disappears in } \\
\text { alkaline } \\
\text { solution } \\
\end{array}$ & $\begin{array}{c}\text { Water, etha- } \\
\text { nol; insoluble } \\
\text { in ether }\end{array}$ & Great & \\
\hline $\begin{array}{l}\text { Quinic acid } \\
\text { Inositol }\end{array}$ & $\begin{array}{l}\text { None } \\
\text { None }\end{array}$ & $\begin{array}{l}\text { Water } \\
\text { Water }\end{array}$ & . & $\begin{array}{l}312 \mathrm{~m} \mu \\
312 \mathrm{~m} \mu\end{array}$ & " & i & : & $\begin{array}{l}p \text {-Benzoqui- } \\
\text { none disul- } \\
\text { phonic acid }\end{array}$ \\
\hline
\end{tabular}

when the solution is made alkaline, and reappears again, when the solution is acidified. The synthetic $p$-benzoquinone disulphonic acid likewise shows the same qualities (Fig. 4). These substances also agree in being very easily further oxidized, even at room temperature. By this reaction the absorption maxima are lost (Table 2).

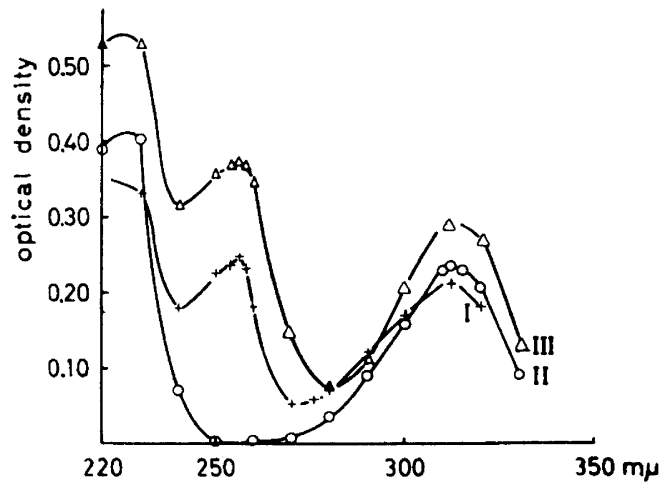

Fig. 3. The absorption in ultra-violet light of the product from hydroquinone and molten potassium bisulphate, I; synthetic hydroquinone disulphonic acid, II; partly oxidized synthetical hydroquinone disulphonic acid, III. Solvent distilled water.

Acta Chem. Scand. 13 (1959) No. 3 
Fig. 4. The pH dependence of the ultraviolet spectrum of quinone disulphonic acid. I, pH about 4; II, $\mathrm{pH}$ about 12; III, $\mathrm{pH}$ restored to 4 . Solvent distilled water.

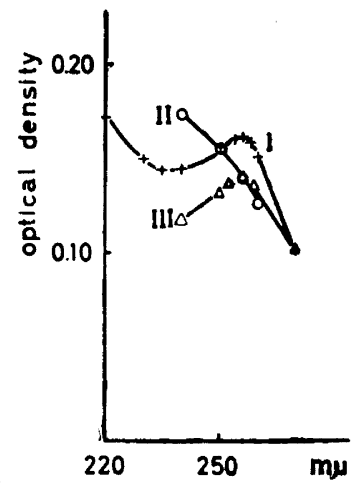

\section{DISCUSSION}

The experiments with the hydroquinone group yield some information of the reactions that can take place in the molten potassium bisulphate. Sulphonation of hydroquinone in this milieu always gives the disulphonic acid. The wellknown dehydration reaction (compare the acrolein formation from glycerol in molten potassium bisulphate) occurs, when inositol and quinic acid are treated with the molten potassium bisulphate. Thus, by this treatment, an aliphatic carbocyclic structure with several hydroxylic groups can thus be transformed into an aromatic one. Sugars do not seem to react in this way (Table 1). The decarboxylation of 2,5-dihydroxybenzoic acid is certainly effected by the rather high temperature in the molten potassium bisulphate.

Of the tested compounds the products from $\alpha$-and $\delta$-tocopherol are most similar to the antagglutin degradation product. Yet it is surprising that both $\alpha$-and $\delta$-tocopherol give the same product, although there are three methyl groups in the benzene nucleus in the former, and only one in the latter substance. Durohydroquinone, that has the same benzene nucleus as $\alpha$ tocopherol, does not give after corresponding treatment any product similar to the degradation product of antagglutin. It is therefore probable that the chromane structure of the $\alpha$-tocopherol is at least partly retained in the product from $\alpha$-tocopherol after heating in molten potassium bisulphate.

The aromatic compound of the male sperm antagglutin has been compared with tocopherols ${ }^{4,5}$, and it has been shown that the derivative extracted from antagglutin is not identical with any of the known tocophercls ${ }^{5}$. It also appears most probable that this aromatic compound is an artefact originating from an aliphatic precursor constituent bound in the antagglutin molecule ${ }^{5}$. In fact, the intact antagglutin has no specific absorption in ultra-violet light like that of aromatic compounds.

The many resemblances between quinone disulphonic acid and the antagglutin degradation product point (Table 2) to a quinone-like structure, but no absorption maximum after reduction related to a hydroquinone has been detected. The situation of the absorption maximum of the antagglutin degradation product at $256 \mathrm{~m} \mu$ in connection with the fact that an oxidation

Acta Chem. Scand. 13 (1959) No. 3 
is necessary in order to obtain this maximum makes it reasonable to propose that the chemical structure underlying the spectrum may be a carbonylic substance with two conjugated ethylenic bounds. The transformation in the molten potassium bisulphate of carbocyclic compounds like quinic acid and inositol into hydroquinone disulphonic acid suggests that the precursor constituent of the antagglutin degradation product and also of the extractable aromatic compound mentioned above is a carbocyclic residue with some hydroxylic groups.

Acknowledgment. The author is very much indebted to Professor P. E. Lindahl for advice and valuable discussions and for reading of the manuscript. This study was aided by grants from the Population Council, New York, and from the Swedish Natural Scienes Research Council.

\section{REFERENCES}

1. Lindahl P. E. and Kihlström J. E. Report of the II International Congress of Physiology and Pathology of Animal Reproduction and of Artificial Insemination, Copenhagen 1952 p. 70.

2. Lindahl P. E. and Kihlström J. E. Fertility and Sterility 5 (1954) 241.

3. Kihlström J. E. Arkiv Kemi 7 (1954) 399.

4. Lindahl P. E. and Kihlström J. E. Nature 174 (1954) 600.

5. Lindahl P. E., Kihlström J. E. and Ross S. Acta Chem. Scand. 10 (1956) 1997.

6. Lindahl P. E., Kihlström J. E. and Ross S. Acta Chem. Scand. 11 (1957) 1.

7. von Hesse, O. Ann. 110 (1956) 194.

Received November 3, 1958. 